\title{
Improved APAC algorithm for minimizing delay in wireless sensor network with mobile sink
}

\author{
Hinal Upadhyay ${ }^{*}$ and Mala Mehta ${ }^{2}$ \\ PG Student, Department of Information Technology, SVIT, Vasad, Gujarat Technological University, Gujarat, \\ India $^{1}$ \\ Assistant Professor, Department of Information Technology, SVIT, Vasad, Gujarat Technological University, \\ Gujarat, India ${ }^{2}$
}

Received: 05-July-2016; Revised: 28-October-2016; Accepted: 16-December-2016

(C)2017 ACCENTS

\begin{abstract}
A wireless sensor network (WSN) is a collection of small randomly dispersed devices. It is denoted as sensor nodes, which can sense the physical or environmental conditions, such as temperature, sound, pressure, etc. and pass their data through the network to a main location. Currently, research is going on in the field of WSN about latency, energy consumption, speed and security. This paper is focused on delivery latency minimization problem (DLMP). To reduce the latency, shortest path must be found. If the travelling path is shorter than latency can be decreased. This research presented improved anchor point algorithm with clustering (APAC) in WSNs. Using better anchor point selection in point substitution method, latency can be minimized. After applying point substitution method, the clustering method with lowenergy adaptive clustering hierarchy (LEACH) protocol has been applied. Results show that latency is reduced compared to the only point substitution method.
\end{abstract}

\section{Keywords}

Wireless sensor network (WSN), Delivery latency minimization problem (DLMP), Clustering, Mobile sink.

\section{Introduction}

A Wireless sensor network is defined as a network of devices which are denoted as nodes, which can sense the environment and communicate the information gathered from the monitored field through wireless links [1]. The data is forwarded, via multiple hops to a sink which uses it locally or is connected to other networks (e.g., the Internet) through a gateway [2-4]. The wireless sensor networks have issues related to latency, speed, security and more energy usage. Recent research shows that latency minimization is a major problem in wireless sensor network [5-9]. For this, different methods and algorithms are available in past research work. In wireless sensor networks, sink works like a gateway. There are different types of sinks like moving sink, static sink, single sink and multiple sink. As per past research, moving sink is better compared to other. One single mobile sink (WSN-MS) provides an effective method to collect data [10-16]. In WSN-MS, all sensor nodes except mobile sink are stationary, and mobile sink can access sensor node's data by moving around them.

\footnotetext{
*Author for correspondence
}

In a WSN-MS, The delivery latency is defined as the Time- mobile sink takes to traverse the sensing range of sensor nodes once to collect data and deliver the collected data to the base station [1]. The main reason for long latency is the long moving time of mobile sink and length of travel route. To minimize the latency, travel route should be shorter.

As per the past research, using clustering algorithms, the result of latency can be improved by using position-based aggregator node election protocol (PANEL) algorithm, LEACH protocol. To reduce the latency, improved APAC Algorithm with mobile sink has been used in this paper. In this algorithm two methods are used. First is point substitution and second is clustering with LEACH protocol.

There are many tools available for WSN like J-Sim, TOSSIM, NS-2, OmNet++ and Castalia. The Castalia simulator can be used in WSN and body area network. Castalia is based on OMNeT++. OMNeT++ is an extensible, modular, component-based $\mathrm{C}++$ simulation library and framework, primarily for building network simulators. Castalia uses $\mathrm{OMNeT}++$ as its base. It is suggested that a fair 
understanding of the basic concepts of OMNeT, is necessary to learn castalia. Castalia tool gives better results compared to other tools.

WSN are spatially distributed autonomous sensors to monitor physical or environmental conditions, such as temperature, sound, pressure, etc. and to cooperatively pass their data through the network to a main location. But WSN has many challenging issues like security, speed, latency and more energy usage. In WSN, the sensor node passes its data to the sink and sink collects the data from all sensor nodes and sends it to the main base station.

A wireless sensor is an embedded system with a communication function and it is capable to measure physical quantities such as heat, humidity, temperature, vibration, radiation, sound, light, movement, etc. It converts them into digital values which are sent as sensed data to a remote processing station or base station [11].

The paper is structured in various sections. The section 1 contains the background and objectives. The section 2 and 3 present the point substitution method and clustering method respectively. Section 4 contains the proposed algorithm. Section 5 contains simulation results. Paper is then concluded by the conclusion, future work and references.

\section{Point substitution method}

The DLMP considered and discussed in WSNs [1]. Authors have proved that the DLMP is an NPcomplete problem. The main goal is to minimize the travel latency of mobile sink. To minimize the travel route, they proposed a substitution heuristic algorithm with point substitution and line substitution method. They considered single mobile sink, which is moving and it collects the data from all sensor nodes at the same time, the sensor nodes are static. Their work can be summarized as follows. First, they formulate the DLMP as an integer programming. Second, it proves that DLMP is an NPC problem, the authors showed that the anchor point should be located at the border of communication range of sensor nodes such that the length of travel route would be shorter. Third, a substitution heuristic algorithm is proposed to plan the travel route of mobile sink by point substitution and line substitution methods and delivery latency is minimized by linear programming. After the simulation, they have compared their results with travelling salesman problem (TSP) heuristic algorithm and random heuristic algorithm which proved that proposed method is better. The point substitution method is based on Anchor point concept.

Anchor point can be defined as:

Definition (Anchor point): Since the travel route of mobile sink is made up of joint line segments, we define the turning points of line segments in terms of anchor points [1].

$\mathrm{A}=\{\mathrm{a} 1, \mathrm{a} 2, \ldots, \mathrm{ak}\}$

Where $\mathrm{A}$ is the set of anchor points with $\mathrm{k}$ elements, and $\mathrm{a} 1, \mathrm{a} 2, \ldots$, ak are $\mathrm{k}$ anchor points.

$\mathrm{A}=\{\mathrm{a} 1, \mathrm{a} 2, \mathrm{a} 3\}$, and the travel route is made up of line segments $\mathrm{s} 0 \mathrm{a} 1, \mathrm{a} 1 \mathrm{a} 2, \mathrm{a} 2 \mathrm{a} 3$, and a $3 \mathrm{~s} 0$.

Theorem 1 (Anchor points on the shortest travel route): If a travel route is the shortest, its anchor points must be at the border of communication range of sensor nodes [1].

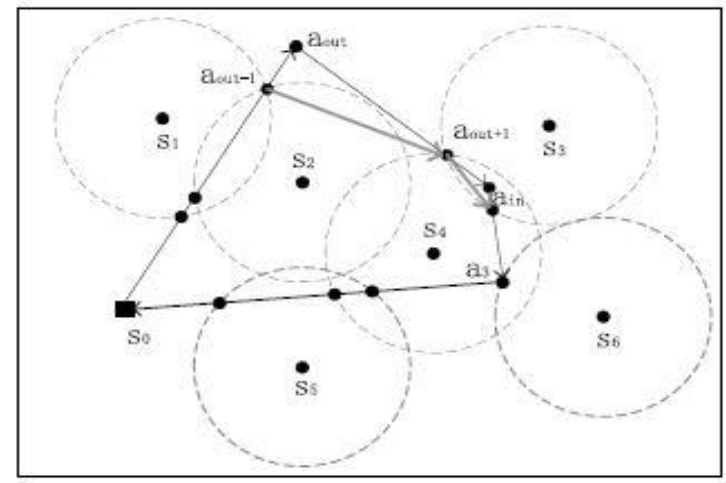

Figure 1 An Illustration of the anchor points

In above Figure $1[1]$, the point $\mathrm{a}_{\text {out }}$ is outside the communication range and $\mathrm{a}_{\mathrm{in}}$ is inside the communication range. To make the travel route shorter, anchor point must be on the border of communication range. So, they replacing the travel segment a $\mathrm{a}_{\text {out }}$ to $1\left(\mathrm{p}\left(\mathrm{a}_{\text {out }}-1\right), \mathrm{p}\left(\mathrm{a}_{\text {out }}\right)\right)$ and $\mathrm{l}(\mathrm{p}$ $\left.\left(\mathrm{a}_{\text {out }}\right), \mathrm{p}\left(\mathrm{a}_{\text {out }}+1\right)\right)$ by the travel segment $1\left(\mathrm{p}\left(\mathrm{a}_{\text {out }}-1\right.\right.$ ),$\left.p\left(a_{\text {out }}+1\right)\right)$ And for $\mathrm{a}_{\text {in }} 1\left(\mathrm{p}\left(\mathrm{a}_{\text {in }}-1\right), \mathrm{p}\left(\mathrm{a}_{\text {in }}\right)\right)$ and 1 $\left(\mathrm{p}\left(\mathrm{a}_{\mathrm{in}}\right), \mathrm{p}\left(\mathrm{a}_{\mathrm{in}}+1\right)\right)$ by the travel segment $\mathrm{l}\left(\mathrm{p}\left(\mathrm{a}_{\mathrm{in}}-\right.\right.$ $\left.1), \mathrm{p}\left(\mathrm{a}_{\mathrm{in}}+1\right)\right)$.

So, using better anchor point selection the travel route becomes shorter.

Point substitution: By theorem 1, when anchor points are located at the border of communication range of sensor nodes, the length of travel route is shorter. Based on this property, the point substitution is to transfer anchor points from the center to the border of communication range of sensor nodes. So, 
using a point substitution method the travel route becomes shorter and latency is also decreased.

\section{Clustering}

Clustering is the process of connecting two or more devices in such a way that they behave like a single device. Clustering is used for parallel processing, load balancing and fault tolerance.

In the previous research works, it is shown that using clustering method, latency can be reduced. In recent research papers, different authors used different methods and different algorithms to reduce latency. There are many different clustering algorithms available. In clustering, all sensor nodes send their data to the cluster head and cluster head sends all the data to the main base station. Cluster head selection is an important process. In [2] authors generalized the multi-parent method to support a multi-cluster model for the network where they assumed that the network had multiple central points called cluster-head $(\mathrm{CH})$ those were in charge of scheduling the nodes in the network. In [3] authors proposed a prediction-based data-aware clustering in wireless sensor networks. They formed steady clusters and so significantly reduced the amount of communication required to report the final results to the sink. LEACH is first energy efficient clustering routing protocol. Many protocols are derived from LEACH with some modification such as two-level LEACH, multi-hop LEACH, E-LEACH, vice-cluster head LEACH, mobile LEACH, advanced LEACH (LEACH-A), balanced LEACH and LEACH-S. In [5] authors proposed low-latency data collection network structure (LDCNS) which minimizes delays in a data collection process. The LDCN organizes sensor nodes into multiple multi-layered clusters. In [6] authors proposed a novel clustering algorithm, frontleading energy efficient cluster heads (FLEECH), in which the whole network is partitioned into regions with diminishing sizes. LEACH divides the wireless sensor network into several clusters. Each cluster has a cluster head that aggregates the data from the cluster nodes and processes the data and transmits it to the base station. LEACH uses a randomize rotation of high-energy $\mathrm{CH}$ position rather than selecting in the fix manner of $\mathrm{CH}$, so that every node has a chance to become a cluster head and we can avoid the battery depletion problem of an individual sensor and quick death, as in direct communication. The number of cluster heads and cluster members generated by LEACH protocol are important parameters for better results. The cluster head makes a time division multiple access (TDMA) schedule for the other sensor nodes under its cluster [7]. The communication between different clusters is done through $\mathrm{CHs}$ in a code division multiple access (CDMA) way. The cluster heads collect the data from their non-cluster head members and aggregate it before sending it to the other $\mathrm{CHs}$ or base station (BS). After a predetermined time lapse, the cluster formation step is repeated so that different nodes are given a chance to become $\mathrm{CHs}$ and energy consumption is thus uniformly distributed [7]. This protocol works mainly in rounds

(1) Advertisement phase

(2) Cluster set-up phase

In advertisement phase, the node decides Independently of other nodes if it will become a cluster head or not. After that, $\mathrm{CH}$ informs their Neighborhood that it has become a cluster head. In cluster set-up phase, other non-cluster head members inform to the cluster head that they are the members of that cluster with "join packets" which contains their IDs Using carrier sense multiple access (CSMA) [7]. After the cluster set-up phase, the cluster head knows the no. of member nodes and their IDs. After that $\mathrm{CH}$ creates a TDMA Schedule and broadcast the TDMA table to other cluster members. After compilation of this two phase, steady state phase start, node sends their data to $\mathrm{CH}$ and $\mathrm{CH}$ aggregates this data and sends it to the base station. LEACH is capable to perform the local aggregation of data in each cluster to reduce the amount of data that is transmitted to the base station. As per the past Survey, it can say that using clustering method the latency can be reduced. Because there is only a cluster head, which can directly transfer the data so it takes less time for mobile sink to traverse the route. If mobile sink takes less time to complete the route than latency is decreased.

\section{Proposed work}

In WSN, when any node sends the data to the sink through the link, it has to find a shortest travel route to minimize the latency. To reduce the latency, clustering method was introduced using different algorithms and the second method is a point substitution method. Using these two methods, travel route can be shorter. To make a travel route shorter, we have to find optimize routing path which can cover all the sensor nodes and the sink collects the data from all sensor nodes and sends this directly to the base station. For that, routing path has been decided before sink starts moving. The problem is that if we can find a nearest anchor point from the sink, then the travel route becomes much shorter. 
Because if travel route becomes shorter, sink can easily collect the data from the sensor node in less time.

To reduce the latency, the new APAC algorithm has been proposed in this paper. The two methods have been combined which are-point substitution and clustering method. Necessary assumptions have been taken for the proposed work. In a Network Scenario, six sensor nodes are taken and these six sensor nodes are stationary. One mobile sink is considered which is moving. When it starts moving, it collects the data from all sensor nodes. We have set the sensor nodes' positions using graphical representation. And Sink knows the entire sensor node's position geographically.
Table 1 shows considered parameters those are taken in the proposed work. Figure 2 shows the normal Scenario with the positions of all sensor nodes and also moving the sink. The values of $s_{0}$ to $s_{6}$ are plotted graphically to have the Exact Situation.

Table 1 Parameters taken

\begin{tabular}{ll}
\hline Parameter & Comments \\
\hline $\mathrm{S}_{1}$ to $\mathrm{S}_{6}$ & Static sensor nodes \\
$\mathrm{S}_{0}$ & Moving sink \\
Communication & $100 \mathrm{~m}$ \\
Radius & \\
Plane deployed for WSN- & {$[0,800] \times[0,600]$} \\
$\mathrm{MS}$ & \\
\hline
\end{tabular}

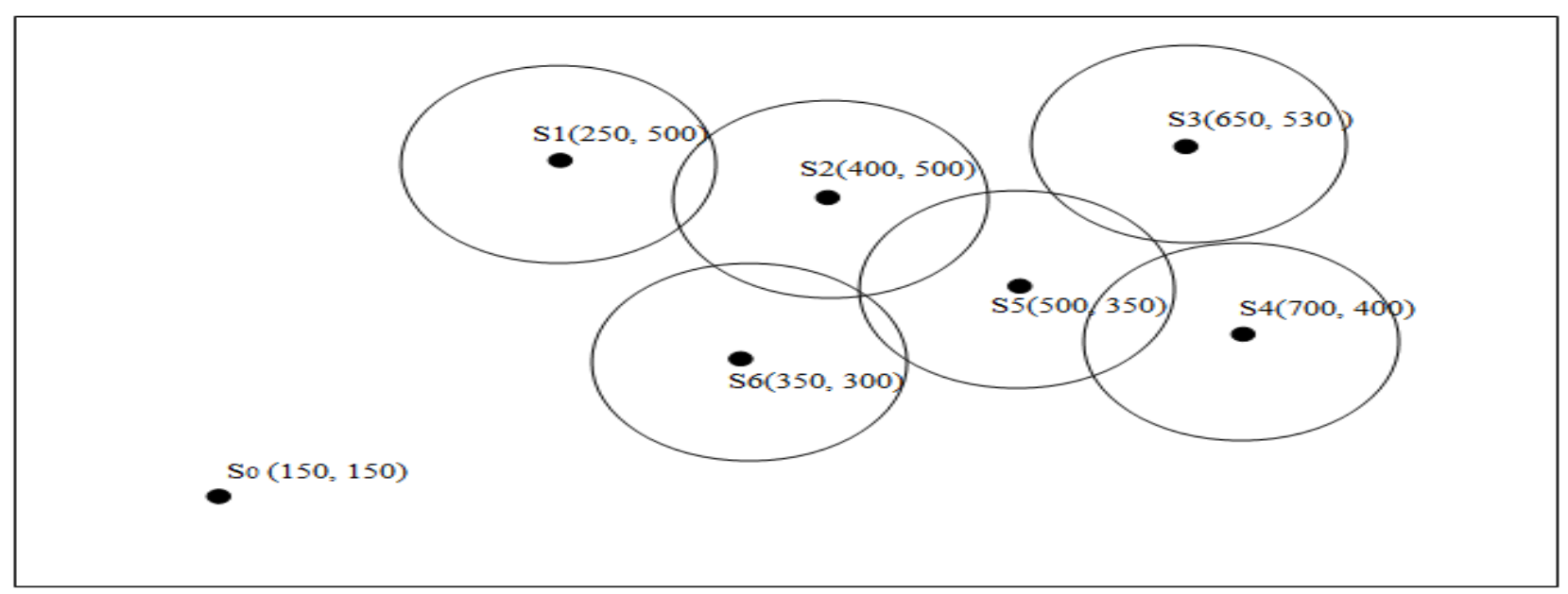

Figure 2 General scenario

\section{Improved APAC algorithm:}

Step 1: Input is taken in the form of coordinates for $\mathrm{S}_{0}$ to $\mathrm{S}_{\mathrm{n}}$. All sensor nodes are static and one mobile sink is moving. Sink moves and collects the data from all sensor nodes in one round.

Step 2: TSP: First TSP algorithm is applied in created scenario and it finds shortest route and visits all nodes at least once and finds out minimum route with shortest route length.

Figure 3 shows the flowchart of the improved APAC algorithm. Figure 4 shows that after applying TSP algorithm, it gives one shortest route with minimum distance and visits all the nodes. It gives Shortest path: 1 -> 7 -> 6 -> 5 -> 4 -> 3 -> 2 -> 1

Step 3: Point substitution method: In point substitution method, the anchor point is decided, which is on the border of communication range. First mathematical formula is applied and all points on the border of communication range are found out.

$\mathrm{x}(\Theta)=\mathrm{rcos}(\Theta)+\mathrm{j}$

$y(\Theta)=r \sin (\Theta)+k$

Where;

$r=$ Radius of circle

$\Theta=$ Angle between 0 to 360

$\mathrm{j}, \mathrm{k}=$ Value of sensor node $\left(\mathrm{s}_{1}\right.$ to $\left.\mathrm{s}_{6}\right)$

$\mathrm{x}, \mathrm{y}=$ points on the circle

Then all points' distances are compared with mobile sink and minimum distance between mobile sink and the point which is in communication range is discovered using a Euclidian distance expression.

$\mathrm{E}=\sqrt{ }\left(\mathrm{x}_{1}-\mathrm{y}_{1}\right)^{2}+\left(\mathrm{x}_{2}-\mathrm{y}_{2}\right)^{2}$

Where $\mathrm{E}=$ Value of Euclidian distance

$\left(\mathrm{x}_{1}, \mathrm{y}_{1}\right)=$ Starting points

$\left(\mathrm{x}_{2}, \mathrm{y}_{2}\right)=$ End points 
These points are considered as Anchor points. Sink starts moving to collect the data from the anchor points. So, after applying point substitution method using Euclidian distance formula, anchor points on the border of communication range was received.

Figure 5 shows that after applying point substitution method, the travel route becomes shorter and we get anchor points $\mathrm{a}_{1}, \mathrm{a}_{2}, \mathrm{a}_{3}, \mathrm{a}_{4}, \mathrm{a}_{5}, \mathrm{a}_{6}$ nearest to the sink node and the mobile sink starts moving and collects the data from the anchor point.

Step 4: Clustering method

After finding out anchor points, clustering method is applied to anchor points. As per previous research sources on clustering algorithms, the LEACH protocol is better compared to other algorithms and it is considered suitable for small scenarios. So, for clustering, LEACH protocol has been used. LEACH is based on an aggregation (or fusion) technique that combines or aggregates the original data into a smaller size of data that carries only meaningful information to all individual sensors. LEACH divides the network into several clusters of sensors and it selects the cluster head randomly every time.

Step 5: Using above method the route length becomes shorter and it minimizes the delay.
Take input $\mathrm{i}=\mathrm{S} 1$ to $\mathrm{Sn}$ Sensor Nodes are static, Mobile sink is moving

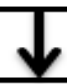

Apply Travelling Salesman Problem (TSP)

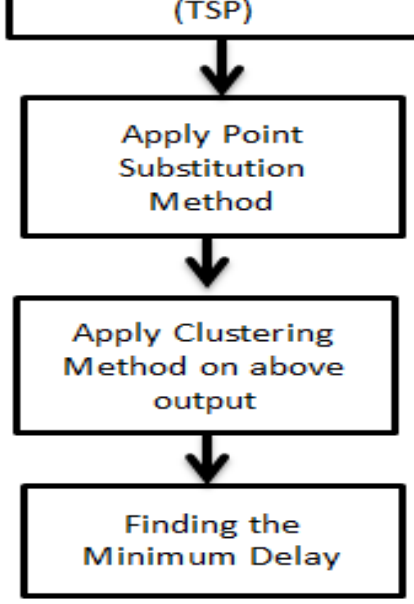

Figure 3 Flowchart of improved APAC algorithm

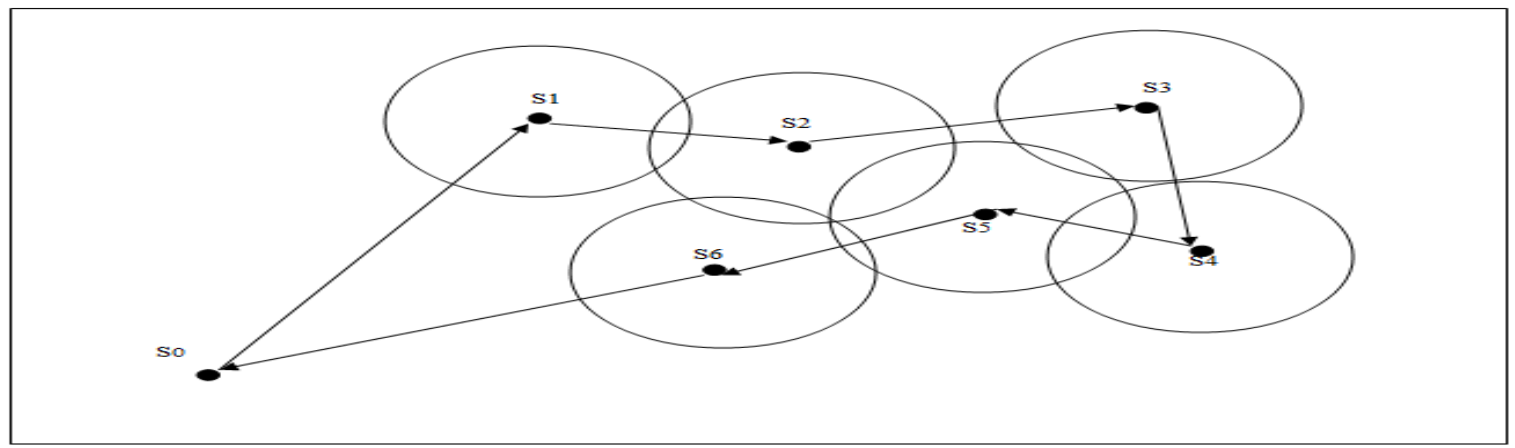

Figure 4 TSP scenario

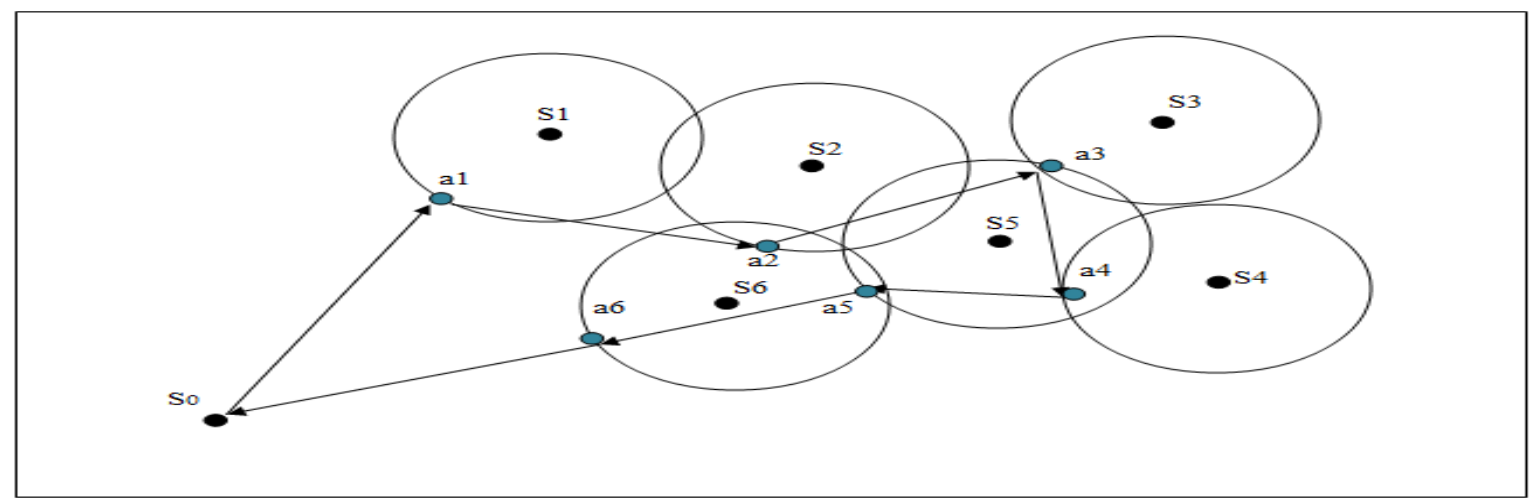

Figure 5 Anchor points scenario 


\section{Simulation results}

Default simulation parameters are taken as shown in Table 1. Figure 4 and Figure 5 shows the travel route obtained by the TSP and a point substitution method respectively. It is observed that the travel route obtained by the point substitution method is shorter than the TSP algorithm. Castalia tool is selected for the simulation. Castalia is a simulator for WSN; body area networks (BANs) and generally for networks of low-power embedded devices. The simulation time taken is 15 seconds for all scenarios. The radius taken is 100 meters. Radius and simulation time can be changed if needed as per scenario. The packets received per node are also increasing in point substitution method compared to TSP.

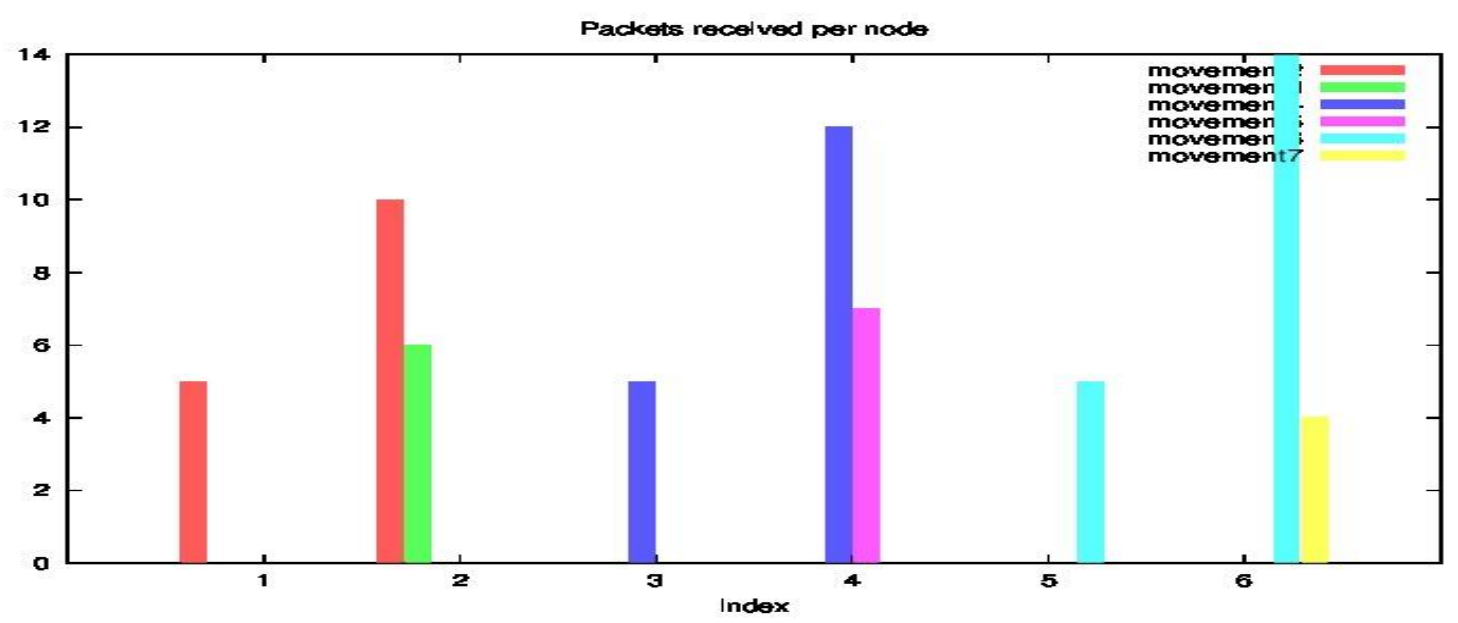

Figure 6 Packets received per node (TSP)

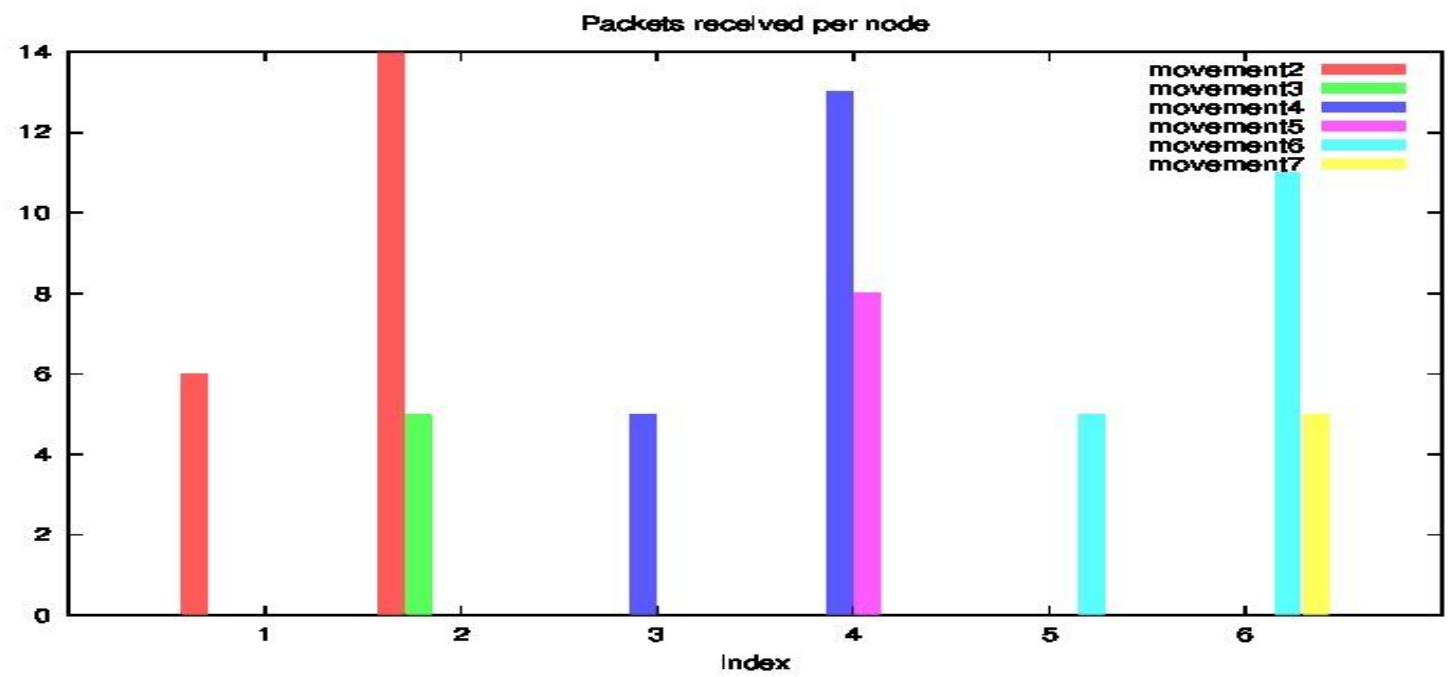

Figure 7 Packets received per node (PS)

Figure 6 presents the packets received per node for TSP and Figure 7 presents the packets received per node for point substitution method. Where $\mathrm{x}$-axis presents sensor nodes and y-axis present number of packets. We can see that more packets are received by a sink in point substitution method. Table 2 shows the comparison between TSP and point substitution (PS) method.
Table 2 Comparison of travelling salesman problem and point substitution method

\begin{tabular}{lll}
\hline Parameters & TSP & PS \\
\hline Route length & 1509.56 & 1343.94 \\
Packets & 68 & 72 \\
Received & & \\
\hline
\end{tabular}




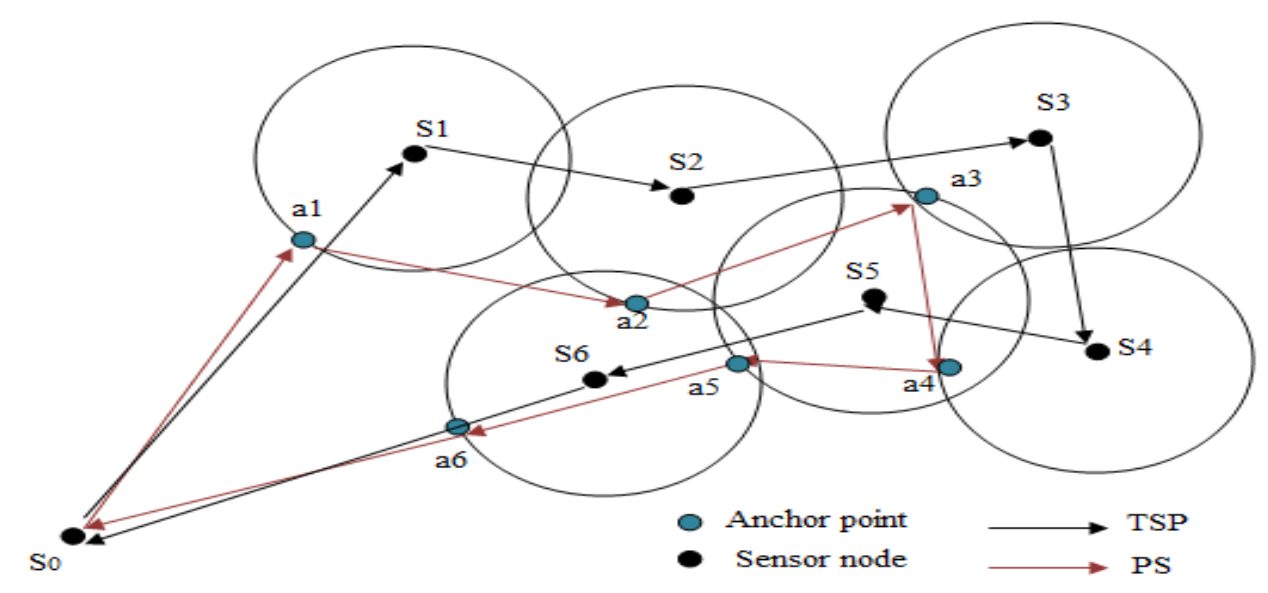

Figure 8 Route comparison between TSP and PS

Table 2 shows the comparison between TSP and PS methods which reflect that, in the PS method more packets are received. In Figure 8, two routes, each for TSP and PS have been shown. After comparison of TSP and PS, it can be concluded that the PS route becomes smaller than the TSP. Table 3, 4, 5 and 6 shows the comparison of TSP and PS for different variations of radius.

\section{Results for different communication radiuses:}

Results for radius 100 meters are derived. Different radius in every simulation can be taken and observations are analyzed. Different anchor points in the different radius selection are considered. We have taken 4 different radiuses for simulation and we can see the results in below table. Also, we can see a different radius comparison for receiving packets by a sink in Figure 9.

Table 3 Comparison of TSP and PS for $70 \mathrm{~m}$

\begin{tabular}{lll}
\hline Parameters & TSP & PS \\
\hline Route length $(\mathrm{m})$ & $1509.56 \mathrm{~m}$ & $1224.51 \mathrm{~m}$ \\
Packets & 52 & 53 \\
Received & & \\
\hline
\end{tabular}

Table 4 Comparison of TSP and PS for $120 \mathrm{~m}$

\begin{tabular}{lll}
\hline Parameters & TSP & PS \\
\hline Route length $(\mathrm{m})$ & $1509.56 \mathrm{~m}$ & $1206.34 \mathrm{~m}$ \\
Packets Received & 98 & 115 \\
\hline
\end{tabular}

Table 5 Comparison of TSP and PS for $150 \mathrm{~m}$

\begin{tabular}{lll}
\hline Parameters & TSP & PS \\
\hline Route length $(\mathrm{m})$ & $1509.56 \mathrm{~m}$ & $1124.31 \mathrm{~m}$ \\
Packets Received & 133 & 206 \\
\hline
\end{tabular}

Table 6 Comparison of TSP and PS for $180 \mathrm{~m}$

\begin{tabular}{lll}
\hline Parameters & TSP & PS \\
\hline Route length $(\mathrm{m})$ & $1509.56 \mathrm{~m}$ & $1040.96 \mathrm{~m}$ \\
Packets Received & 161 & 248 \\
\hline
\end{tabular}

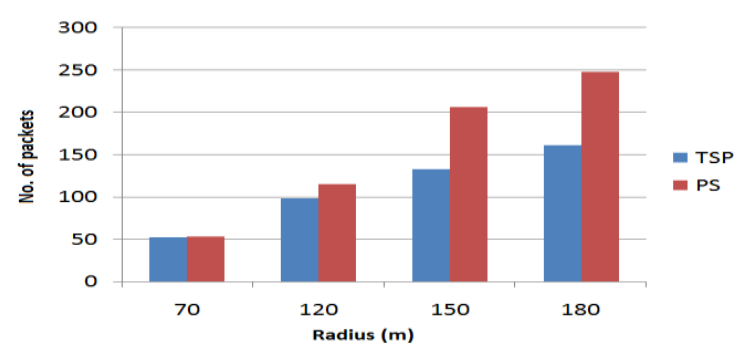

Figure 9 Comparison of different radius for received packets

\section{LEACH Protocol}

For leach protocol, another small scenario is taken. Following scenario is considered,

Simulation time $=50$ seconds

Area $=50 * 50$ meters

Sensor nodes $=7$ (One mobile sink node and six static nodes)

After applying leach protocol the latency has been decreased compared to PS method. In PS method the latency result found between $[60,80) \mathrm{ms}$ and merging leach protocol with PS method, result found is between $[0,20) \mathrm{ms}$. After applying leach protocol with PS method, meaning that according to our proposed algorithm, if first anchor points are found and then leach protocol is applied then latency decreases. 
It means that merging these two methods for whole scenario gives less latency. Results show that improved APAC is better compared to the only PS method because latency is decreased in new proposed method compared to the only point substitution method. Increase in simulation time shows that more packets are received in both the cases and latency results remain same. Same latency interval received when the simulation time was increased. The Figure 10, 11 and 12 present the result of the only PS method, our proposed algorithm result and comparison of PS and our proposed algorithm respectively.

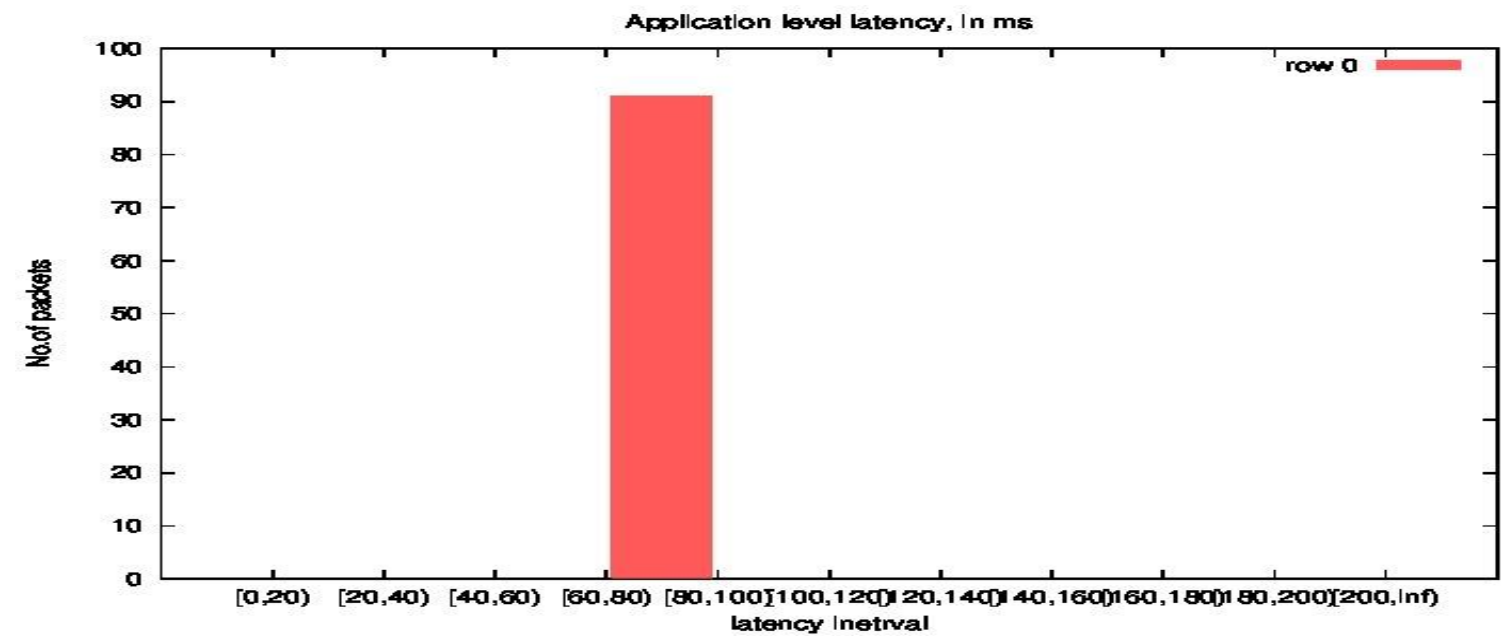

Figure 10 Latency result for point substitution method

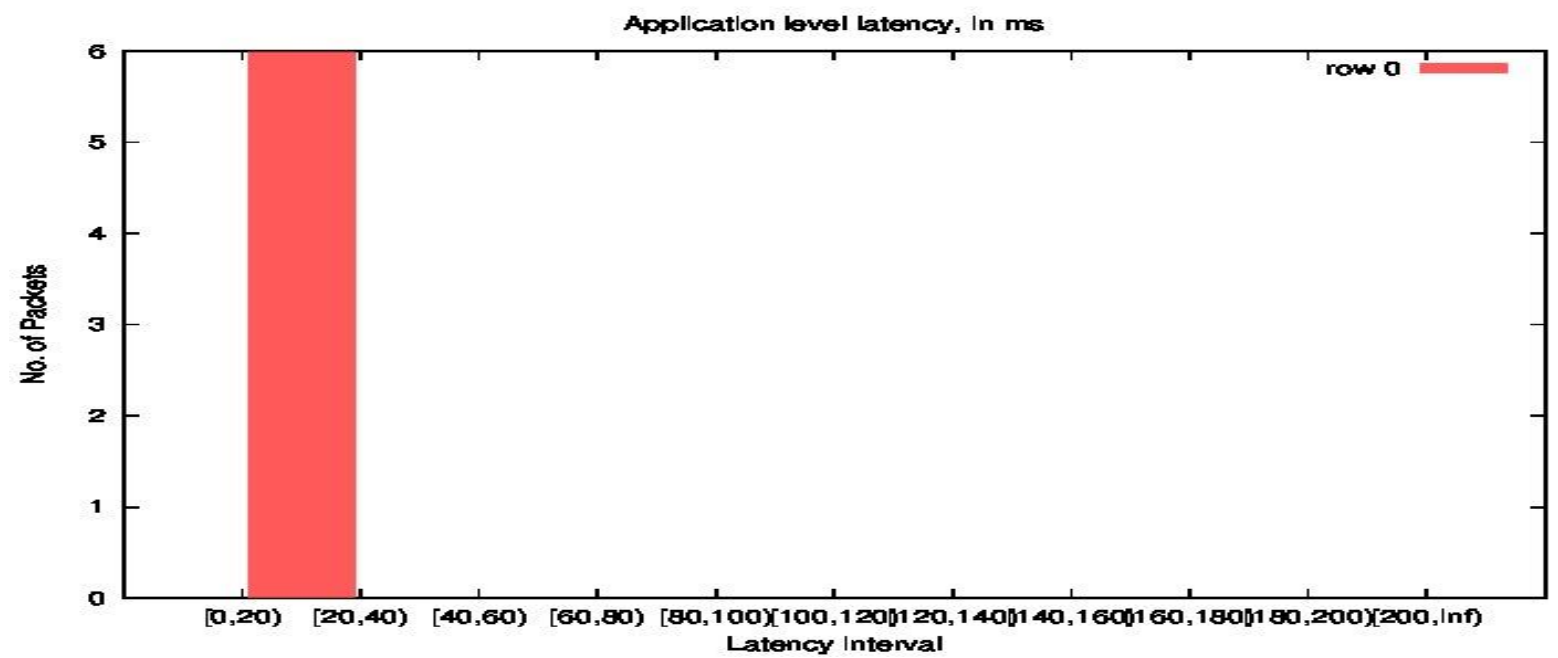

Figure 11 Latency result for leach protocol

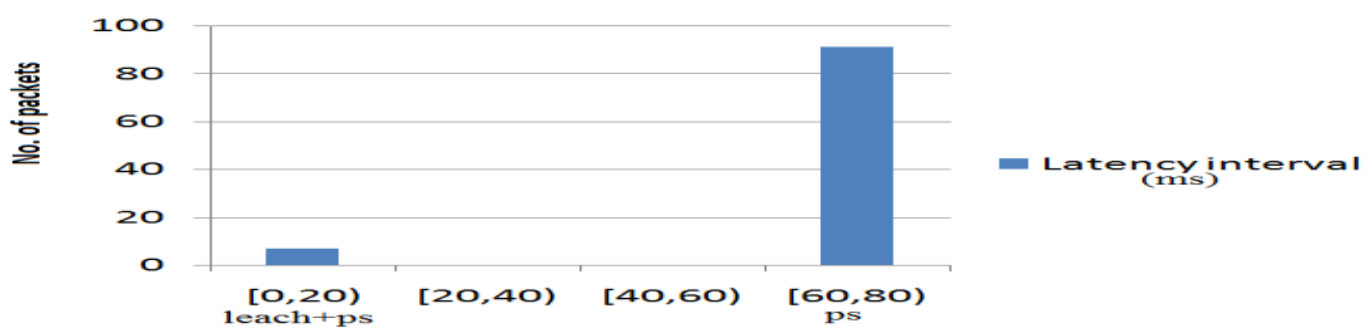

Figure 12 Comparison graph for leach and PS method 


\section{Conclusion and future work}

It has been concluded that using improved APAC algorithm latency is minimized. Point substitution method is applied over TSP and results show that more packets are received, the route length becomes shorter. After applying clustering method (LEACH protocol) on anchor points the latency is reduced compared to the only PS method. In the future, better anchor point selection and using different clustering methods the results improved.

\section{Acknowledgment}

None.

\section{Conflicts of interest}

The authors have no conflicts of interest to declare.

\section{References}

[1] Tang J, Guo S, Yang Y. Delivery latency minimization in wireless sensor networks with mobile sink. In IEEE international conference on communications (ICC) 2015 (pp. 6481-6). IEEE.

[2] Lee H, Keshavarzian A, Aghajan H. Multi-cluster multi-parent wake-up scheduling in delay-sensitive wireless sensor networks. In IEEE global telecommunications conference 2008 (pp. 1-6). IEEE.

[3] Ashouri M, Yousefi H, Basiri J, Hemmatyar AM, Movaghar A. PDC: prediction-based data-aware clustering in wireless sensor networks. Journal of Parallel and Distributed Computing. 2015;81:24-35.

[4] Xu R, Dai H, Wang F, Jia Z. A convex hull based optimization to reduce the data delivery latency of the mobile elements in wireless sensor networks. In IEEE international conference on embedded and ubiquitous computing high performance computing and communications 2013 (pp. 2245-52). IEEE.

[5] You X, Xu P, Zhu W. Low-latency energy-balanced data collection in wireless sensor networks. In sixth international conference on wireless communications and signal processing (WCSP) 2014 (pp. 1-6). IEEE.

[6] Nayak BK, Mishra M, Rai SC, Pradhan SK. A novel cluster head selection method for energy efficient wireless sensor network. In international conference on information technology (ICIT) 2014 (pp. 53-7). IEEE.

[7] Vivekchandran KC, Narayan NP. Energy efficiency and latency improving in wireless sensor networks. International Journal of Science and Research. 2015; 4(5):1291-5.

[8] Kaur A, Grover A. LEACH and extended LEACH protocols in wireless sensor network-a survey. International Journal of Computer Applications. 2015; 116(10):1-5.

[9] Martincic F, Schwiebert L. Introduction to wireless sensor networking. John Wiley \& Sons; 2005.

[10] Hamida EB, Chelius G. Strategies for data dissemination to mobile sinks in wireless sensor networks. IEEE Wireless Communications. 2008;15(6):31-7.

[11] Ari AA, Gueroui A, Labraoui N, Yenke BO. Concepts and evolution of research in the field of wireless sensor networks. International Journal of Computer Networks \& Communications. 2015;7(1):81-98.

[12] Buratti C, Conti A, Dardari D, Verdone R. An overview on wireless sensor networks technology and evolution. Sensors. 2009;9(9):6869-96.

[13] Pandey S, Gupta N, Dubey AK. A novel wireless heterogeneous data mining (WHDM) environment based on mobile computing environments. In international conference on communication systems and network technologies (CSNT) 2011 (pp. 298-302). IEEE.

[14] Boulis A. Castalia: A simulator for wireless sensor networks and body area networks. NICTA: National ICT Australia. 2011.

[15] Egea-Lopez E, Vales-Alonso J, Martinez-Sala AS, Pavon-Marino P, García-Haro J. Simulation tools for wireless sensor networks. In proceedings of the international symposium on performance evaluation of computer and telecommunication systems (SPECTS05) 2005 (pp. 2-9).

[16] Xiangning F, Yulin S. Improvement on LEACH protocol of wireless sensor network. In international conference on sensor technologies and applications 2007 (pp. 260-4). IEEE.

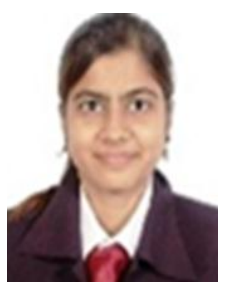

Hinal D. Upadhyay is a PG student at Sardar Vallabhbhai Patel Institute of Technology, Vasad. She has completed her Bachelors of Engineering in Computer Engineering with distinction from Gujarat Technological University in 2014. She is pursuing her masters of Engineering in Computer Engineering from Gujarat Technological University at SVIT vasad. She has published a review paper in $2^{\text {nd }}$ National Conference on Computer \& Communication Research $\left(\mathrm{NC}^{3} \mathrm{R}\right)$ held at VIER college Vadodara.

Email: pretty.sonu1910@gmail.com

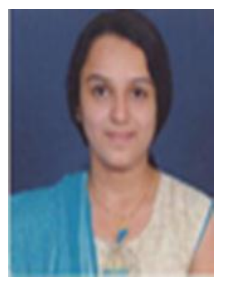

Mala H. Mehta is an Assistant Professor at Sardar Vallabhbhai Patel Institute of Technology, Vasad. She has completed her Bachelors of Engineering in Information Technology with distinction from Gujarat University in 2005. She has completed her Masters of Engineering in Computer Engineering from Gujarat Technological University in 2012 with 9.08 CPI. She has published eleven research papers in various national and international conferences as well as journals. She was given "Young Investigator Award" at an international conference as well. 